\title{
Stratum Spinosum
}

National Cancer Institute

\section{Source}

National Cancer Institute. Stratum Spinosum. NCI Thesaurus. Code C12850.

The part of the epidermis layer of the skin containing Langerhans cells, which are dendritic, MHC class II-expressing antigen presenting cells. 